\title{
A Study on Workflow Resource Management Based on Workflow Net and Agent
}

\author{
Xiang Chen and Xinglin Li \\ School of Management and Economics, Beijing Institute of Technology, Beijing \\ 100081, P.R. China chengxiang@bit.edu.cn 1xl_1022@sohu.com
}

\begin{abstract}
At present, the resource modeling of workflow net (WF-net) are too simple to describe complex workflow. Thus, its application is not satisfying in practical use. Based on the analysis of resource management problems, unified resources management which gets the identical resource request and release process is proposed. The method of identical resource-modeling reduces complexity of WF-net model. Based on colored Petri net, this paper proposes colored WF-net, which is easy to control and computerize. Furthermore, the design of unified resources manage is carried on using the Agent technology, and a method that transforms process definition of WF-net into abstract structure of Agent is produced. By using the appliance character of Agent technology, the method also provides feasibility for unified resources management.
\end{abstract}

Keywords: Workflow net (WF-net), Workflow model, Colored Petri net, Agent, Workflow resource management, Business process analysis

\section{INTRODUCTION}

As a key technology for modern enterprises to realize the process management and process control, workflow provides an integrated framework of modeling, management and operation for enterprise business process [1]. Currently, there are a variety of technologies can be used for workflow modeling, and the workflow net (WF-net) theory based on Petri net[2]is one of them. This theory describes and analyzes complex workflow system model from the perspective of process [3, 4], but the model it built is insufficient in resource management, expression ability and application. SchoSmig and Gong Shi-hao and others established business process model based on Colored Petri net $[5,6]$. This model overcame the problem of the expression ability, but can not use the method of performance analysis and structural analysis related with WF-net. Needs to be pointed out is that the current workflow models based on Petri net neglect the modeling of resource_-an important dimension in workflow, so it will be very difficult to be applied in practical application. In the application aspect, because of independence, cooperation, communication and consultation, ratiocination and other abilities and characteristics [7], Agent is more and more used in workflow management system [8]. However, Agent-based workflow system has deficiencies in process description and inspection of process structure. Based on the research results above, through analyzing the problems of workflow model based on Petri net, we can see that resource Modeling

Please use the following format when citing this chapter:

Chen, X., I i, X., 2007, in IFIP International Federation for Information Processing, Volume 254. Research and Practical Issues of Enterprise Information Systems Il Volume 1, eds. L. Xu. Tjoa A., Chaudhry S. (Boston: Springer), pp. 377. 387 . 
can be adopted to solve the problems of the traditional WF-net. Further, this paper presents the uniform resource management and colored WF-net. Finally, this paper introduces Agent technology into the workflow resource management to enhance existing workflow management system function, and it also discusses the workflow modeling systems based on Petri net and the realization project of Agent-based resource management, ordering to eliminate the gap of the theory and practice and to pave the way for practical application.

\section{WORKFLOW NET RESOURCES MODELING}

WF-net $[6,2]$ presented by Aalst and others is the workflow model based on Petri net, which is studied extensively now. It builds workflow model by mapping the workflow reference model to the Petri net, and uses transition represent activity, place represent the condition which fires activity (if activity need to use some resources), and token represent case to build Petri net model of workflow.

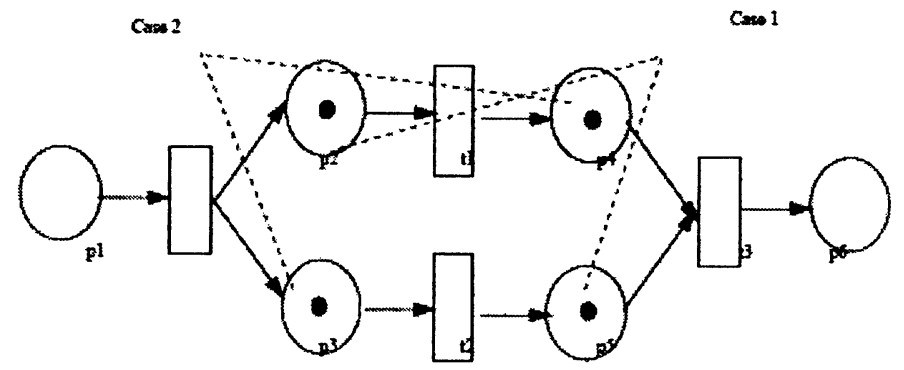

Fignre 1. Example of Handing Multi-cases

This traditional WF-net is legible in description of the static workflow model, but in the control of operation process, especially when multi-cases are in the WF-net, there are some problems. Firstly, the workflow model in the course of operation needs to resolve two problems, one is path selection of the uncertainty activity, and another is application for the roles in the resource manager. Both of two problems in the actual operation course of workflow are dynamic, and the traditional WF-net has been unable to resolve them. Secondly, the workflow model in the course of operation needs to run multiple cases, and an instance may appear that two cases start and run two parallel activities simultaneously. As shown in figure 1, Workflow needs to handle a number of cases at one time, and each case has one or more tokens in the model, if considering the delay in the actual system, all tokens will mix together and come into confusion. For example, case 1 and case 2 have access to the model successively. Case 1 did not reach $\mathrm{p} 4$ because of the delay $\mathrm{t} 1$, but have arrived $\mathrm{p} 5$ via t2. Meanwhile, case 2 arrived p3, p4, at this time T4 was in activated state, if consuming token is according to first-in first-out principle, or in accordance with the random principle, then there could be a situation that token of case 1 in p5 is consumed and the token of case 2 in $\mathrm{p} 4$ fires $\mathrm{t} 3$, all appearance, it is not wish to see this result in the model. Thirdly, in the traditional WF-net model, a state or condition of each object is expressed as a place, and each change or event is express as a 
transition, but the dynamic character of workflow determines that it has a larger state space. This will make practical application system has more crunodes, which will limit model to be computerized.

In fact, the problem of path selection can be attributed to that: choosing different path is according to different resources. Problem of disordered cases and the large state space can be summed up as that: how to distinguish the states of resources in cases. Thus, the key to solve the problems above is to solve problems of management and resource modeling in the model.

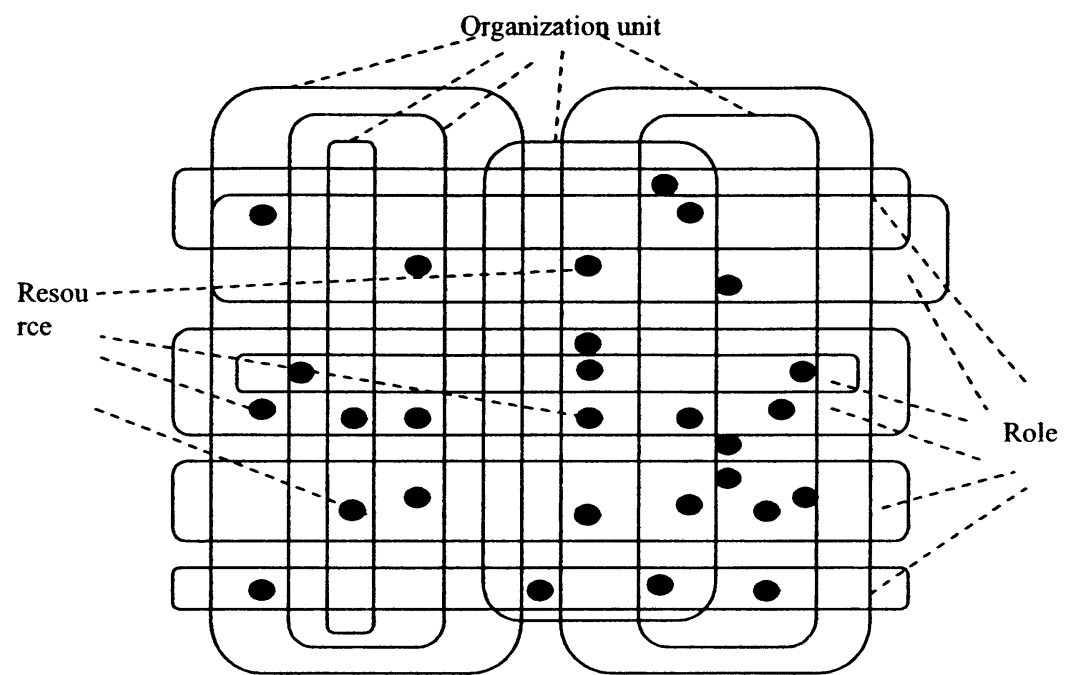

Figure 2. Relation of Organization Unit, Role and Resource

Resource Modeling is the static management of resource information, which is basis of resource allocation in the workflow operation environment. Resource is the implementing entities of task. It is used to analyze composition of Workflow from two logic levels: task logic and organization logic. The former logic reflects the handling process of task the process definition mentioned above. The latter one reflects the organization structure and relationship inside enterprise. These two logic levels integrate together through the organization strategy. This strategy mechanism is in short that: "which must be executed by whom". It shields the realization of behavior activities, and makes the change of flow divide from individual behavior change, thus enhancing the distribution, flexibility and virtual degree of workflow process.

Resource can be classified in accordance with the function itself, the same type such as printing devices and checkers could be put in one organizational unit, and completes the task by resources which are obtained from the resource class by roles, thus, the resource share will be realized between roles in the model, which increases the flexibility of the model. Moreover, the roles and organization units will organize 
resources as the orthogonal relationship as shown in Figure 2. Because the activity is fulfilled by the role, therefore, the examination of initiatory state of each activity is to be the fire mechanism of application for roles, and the completion of activity as the fire mechanism of role demobilization. In this way, a whole process of demand of resources is found in the model, from task execution, to role application, to resource distribution, to role demobilization and then to resource release.

Currently, majority of the workflow models integrate organizational factors in the flow. But according to the above analysis, flow should be three-dimensional structure, which contains participant, role and the process. However, process is based on the cases, it is not very suitable to regard resources as a part of the flow. Therefore, we can consider dividing resources from the process as an independent part, which is unified resource manager for resource management. Thus, flow can be seen as the client port in the client / server architecture, which proposes resource request to the server port_—_ resource manager in the course of checking the initiatory conditions of activity, and determines whether to begin activities according to whether resource manager returns resources or not.



Figure 3. Processes of Resource Request and Release

We build resource manager model for representing the relationship above, as shown in figure 3. Every activity in WF-net will request and release resources through resource manager. When starts an activity, at beginning, PB proposes resource requests to TR_- the resource manager application interface, and the request outputs token to the resource request place for firing through TR - the fire transition. Resource manager allocates appropriate resources by the resource allocation arithmetic, and these resources access to the public interface $\mathrm{RI}$ in the form of token. Activity TA gains resource tokens from RI, these tokens start and complete activities, which will release resources to $\mathrm{RO}$-resource token collection interface, and recover by unified resource manager at last. For each activity in the model, the 
process of using, releasing and accessing to resources is consistent, and they only exchange data with three public interfaces, so it is not necessary to describe them in the model.

Dividing the resource modeling from the WF-net reduces its complexity, and compared with the models based on Petri net other scholars established, this model has a better corresponding relationship with the actual workflow, it is more simple and easier to understand, furthermore, it simplifies the model, which makes the model more easily computerized.

\section{COLORED WF-NET}

Based on the advantages of expression capacity of Colored Petri net, we can endue WF-net that has a basic flow structure with attribute by coloring the WF-net, making it express more meanings, easier to achieve a feature-rich analysis and application software through computer.

Therefore, the token color set $C(p)$ defined in place $p$ represents the attribute set of cases in the state of $p$. This set can be divided into two parts. One is called parameter information, which is for recording operating results and operating process control. Parameter is set as case number, activity types can be fired, type and number of resource requirement and other execution attributes of task. Record of case number possesses the operation identifier of the case in work flow. This identifier is the only one to distinguish cases. Activity type can be fired could record that which transition type can be fired by the place at current state, for the path choosing. Resource type is description of the resource. Another part is called description information, it is used to distinguish different objects, such as contract number, contract type and the latest completion dates of a contract and so on, which can be handled in different way according to the different workflow process and cases.

Token color set $C(t)$ defined on transition $t$ represents the color set that $\exists p \in \epsilon^{*} t, C(p)_{M S}$ must include when $t$ could be fired. It is generally composed by three colors: case number, type and number of resource requirement and activity type. The meaning of the first two colors are consistent with the meaning in the place, activity type designates the category of the activity.

Increasing these colors can enhance interpretation capacity of the original model, rich meaning of model, and increase demands of workflow activity and state transfer modeling. Because color parameters can be added appropriately according to different models, therefore the expansible capacity of model strengthens as well. Based on process operation control in the color definition, it can resolve the problem of uncertainty activity modeling and resource application / release by defined a negative / positive correlation function. Defines the color set resolving these two problems as:

$$
\begin{aligned}
& \text { Jobs }=\left\{J_{1}, J_{2}, \ldots, J_{J}\right\} \quad \text { Activity type } \\
& N O=\{1,2, \ldots \ldots . n\} \quad \text { Case number }
\end{aligned}
$$


$R S=\left\{R_{1}, R_{2}, \ldots, R_{M}\right\} \quad$ Resource requirement type

Defines the color combination as (see the sigh of place and transition as shown in figure 2):

$C(P B)=J o b s \times N O \times R S$ Color combination of activity in initiatory state, $R S$ is resource needed.

$C(P E)=J o b s \times N O \quad$ Color combination of activity after releasing resource at termination condition

$C(T A)=J o b s \times N O \times R S$ Color combination of activity (transition)

$C(R I)=J o b s \times N O \times R S \quad$ Resource applied from resource manager

Define $R=\left\{R_{1}, R_{2}, \ldots R_{R}\right\}$ as set of roles in resource manager, so negative / positive correlation function is defined as:

$$
\begin{aligned}
& \forall R_{r}, R_{s} \in R, \forall J_{k}, J_{l} \in J o b s, \forall N_{n}, N_{m} \in N O:
\end{aligned}
$$



$$
\begin{aligned}
& I_{+}(R I, T A)\left(\left(J_{k}, N_{m}, R_{s}\right),\left(J_{l}, N_{n}, R_{r}\right)\right)=\left\{\begin{array}{c}
1 \text { if } J_{k}=J_{l}, N_{m}=N_{n}, R_{r}=R_{s} \\
0
\end{array}\right. \\
& I_{+}(P B, T A)=I_{|C(P B)|}=I_{|C(T A)|}=\left(\begin{array}{lll}
1 & & 0 \\
& \ldots & \\
0 & & 1
\end{array}\right)
\end{aligned}
$$




$$
\begin{aligned}
& I_{-}(R O, T A)\left(R_{r},\left(J_{k}, N_{m}, R_{s}\right)\right)=\left\{\begin{array}{cc}
1 & \text { if } \quad R_{r}=R_{s} \\
0 & \text { then }
\end{array}\right. \\
& I_{-}(P E, T A)=I_{|C(P E)|}=I_{|C(T A)|}=\left(\begin{array}{lll}
1 & & 0 \\
& \ldots & \\
0 & & 1
\end{array}\right)
\end{aligned}
$$

Formula 1 represents that model applies resources from resource manager, which are required by the initiatory conditions. Formula 2 represents that the activity could be executed when there are conformable resources in resource manager for the case and activity. Formula 3 represents that the set of resources initiatory condition need to apply is consistent with the set of resources that is needed by transition. Formula 4 represents that activities release resources to resource manager, the released resources must be the ones using when activities were executed. This will solve the role application / release problem. Formula 5 represents that the set of releasing resources in terminated conditions is consistent with the set of resources used by transition. One side, Formula 2 and 3 shows that tokens of the various places in the initiatory state must be in the same case number and the same activity type, only in that way, they can work together to fire the transition, which would resolve the chaotic situation when process multi-cases. The other side, at the initiatory state color Jobs choose the activity could be fired, and all places linked with the activity must choose the type of activities consistent with it, thus, the activity can be fired. This way resolves the problem of the modeling of the uncertainty activities.

Here, define the WF-net add to colors as the colored WF-net:

Definition 1 An Colored WF-net is a seven-tuples

$$
W=\left(P, T, F, C, I_{-}, I_{+}, i\right):
$$

1) $P, T, F, i$ accord with the definition of WF-net $[3,6]$;

2) $C, I_{-}, I_{+}$in the precondition of definition of colored Petri net, its control part is as noted above.

\section{AGENT-BASED COLORED WF-NET RESOURCE MANAGEMENT}

As a groupware system, Workflow Management System has some similar characteristics with the Agent system. They are all distributed cooperative work 
system, and all need to have the self-adaptive and self-automatic and other characteristics. Therefore, the Agent system is very fit for workflow management system, in particular the design of distributed workflow control structure.

In application, using Agent can effectively solve resources confliction, mode petrifaction in execution and other issues in workflow. Therefore, we can use Agent technology to establish a unified resource manager. In the Agent-based workflow management, each resource in organization structure (including person, equipment and database, etc.) is in connection with an Agent.

According to different functions Agent achieves, it can be divided into the following three categories:

Task Agent: Its main function is to explain the workflow process definition, operation states of cases in control process.

Resource Agent: It is connected with the resources to be allocated in workflow.

Role disclaimer Agent: It is for organization, and it ensures to make reasonable arrangements for human resources, constitute the best mode of appointment of personnel.

According to the assume based on separation of resource management and process management, the further work is to transform WF-net process definition and execution process into the abstract structure Agent can cognize, and complete coordination and control of resources in workflow through the interaction, response and coordination mechanism of Agent. Firstly defines the Agent in unified resource manager.

Definitions 2 Agent in resource manager is formally to represent as a entity descript by a five- tuples set, namely Agent $=\langle N, I, E, A c, K>$, of which:

1) $N$ is the unique name or logo of Agent and can describe the type of Agent.

2) $I$ is interface definition of Agent, which descript human-computer interface and communication interface(including communication protocol and $\mathrm{V} / \mathrm{O}$ ) shown in front of the user, generally following the principle that interface separates with the Agent function.

3) $E=\left\{e, e^{\prime}, \ldots\right\}$ is the state set of Agent, which describes internal state of Agent, behaviors of Agent is actually the process transferred from one state to another state.

4) $A c=\left\{\alpha, \alpha^{\prime}, \ldots\right\}$ is Services set of Agent, defining behaviors , embodying the functions, and containing reasoning mechanism of Agent.

5) $K$ is knowledge origin of Agent, describing knowledge, data, inference rules and the reflective resources behaviors of Agent needed, such as data structure, database (and Knowledge Base), etc.

On the state $E=\left\{e, e^{\prime}, \ldots\right\}$ of execution of the workflow, it is the limited set of the discrete and instantaneous state. $A c=\left\{\alpha, \alpha^{\prime}, \ldots\right\}$ is the (limited) action set. WFnet uses $P$ short for place and $T$ short for transition. Therefore, the state set $E$ of Agent and $P$ can establish one-to-one correspondence, namely: $E \Leftrightarrow P$. The same as the action set $A c$ of Agent and $T$, namely: $A c \Leftrightarrow T$.

The basic model of Agent action is as follows, starting from a certain state, Agent chooses an action to act on the state. The result of action is a certain state the 
environment may reach. However, only one state can be truly realized, of course, Agent does not know in advance which state will be realized. Base on the second state, Agent keeps on choosing an action to execute, which may reach a centralized state. Then, Agent will choose another action, so continue. Therefore, an execution $r$ of Agent is a sequence that the state and action are alternate:

$$
r: e_{0} \stackrel{\alpha_{0}}{\longrightarrow} e_{1} \stackrel{\alpha_{1}}{\longrightarrow} e_{2} \stackrel{\alpha_{2}}{\longrightarrow} e_{3} \stackrel{\alpha_{3}}{\longrightarrow} \ldots \stackrel{\alpha_{u-1}}{\longrightarrow} e_{u}
$$

The action model of Agent has similar characteristics with fire sequence used as WF-net model, and mapping relations can be established between them. Presumed that $R$ is finite sequences set of all possibility, and then can think that $R$ has corresponding mapping relationship with coverable tree of WF-net.

For the point that how $A c$ define the action of Agent, assume that $R^{E}$ is the subset of $R$ composed by sequence with end state, import a Agent model, which represents a function, mapping an execution to the action:

$$
A g: R^{E} \rightarrow A C
$$

Thus, Agent decides which action will be executed according to the current system situation. For resources management:

Definitions 3: Agent $\mathrm{Ag}$ is seen as a three-tuples set , $A g=<$ see, action, next $>$, Of which :

see $: E \rightarrow P e r$, which maps a state to an apperceive. Apperceive of workflow comes from that whether there is a corresponding role to execute activity, and obtains adequate resources, which can be acquired through cooperating with the Resource Agent and the Role disclaimer Agent. Reflecting to the WF-net, it is that the corresponding place gets or losts the corresponding token and it takes the function as the mapping of mark see $: P \rightarrow I N$. For example, place $n$ gets token on $M^{\prime}$, so $M^{\prime}\left(p_{n}\right)=M^{\prime}\left(p_{n}\right)+1$.

action $I \rightarrow A c, I$ is the set of all states inside Agent, action defines the mapping from internal states to actions. For WF-net, $I$ can be thought as the current mark, and the mapping from state to action can be defined as fire rule, namely action $: M \rightarrow M^{\prime}$, defined as $M^{\prime}=M+N \vec{\sigma}, i f \exists \sigma, M^{\prime}, M\left[\sigma>M^{\prime}\right.$, of which $\sigma$ is transition sequence.

next $: I \times P$ er $\rightarrow I$, achieve the mapping from the internal states to their perception. It is still the change of mark for WF-net, namely next $: M \times P \rightarrow M$.

Agent behavior can be summarized as follows:

Agent begins with a certain initial internal state $i_{0}$, observes the environment $e$, and has a perception see $(e)$, then updates the internal state of Agent through the next function, finally turns into $n \operatorname{ext}\left(i_{0}, \operatorname{see}(e)\right)$. Agent chooses actions by action $\left(n \operatorname{ext}\left(i_{0}, \operatorname{see}(e)\right)\right)$. Working out this action makes Agent enter anther circle, keeping on apperceiving world outside via see, updating states by next , choosing actions to implement through action. 
Other Agent reasoning mechanism, the application of knowledge and interface definition is unique characteristics of intelligent and interactive of Agent, which WFnet doesn't have, and that is the main reason why introduced the Agent formally also. Existing research results of Agent can be continued to use. Thus, we establish mapping of the main elements in basic Petri net and the abstract structure of Agent, so that Agent can cognize the process definition and execution process of WF-net.

\section{CONCLUSIONS}

This paper focus on solving problems of resource management modeling and application in workflow, and divides resource management from the WF-net model as a uniform resource manager, reduces the difficulty of establishing WF-net modeling, increases the readability of the WF-net. Colored Petri net is introduced to enhance the expression of the model, and solves problem that in the actual operation control, the basic model is difficult to decide dynamic path and may arise the confusion when deal with a number of cases at the same time, which makes model has a very strong adaptability. Meanwhile, this model includes an integrated set of concept, a corresponding representation and the necessary rules, which provide a more comprehensive model framework for expressing workflow abstractly. Based on the model, transforms process definition and execution process of WF-net into the abstract structure that Agent can cognize, enables unified resource manager can be combined with Agent, eliminated contradiction of the theory and application. However, the establishment of workflow model also involves the problems such as data sharing and performance analysis, etc. These are pending further study.

\section{ACKNOWLEDGEMENTS}

Foundation item: Project supported by the National Natural Science Foundation, China (Grant No. 70502021)

\section{REFERENCES}

1. Workflow Management Coalition (John Wiley and Sons: New York, 1997).

2. W.M.P. Van Der Aalst, Three Good reasons for Using a Petri-net-based Workflow Management System, Information and Process Integration in Enterprises: Rethinking documents, eds. T. Wakayama (Kluwer Academic Publishers: Norwell, 1998), pp.161182.

3. X. Chen and G. Xia, Workflow modeling based on colored Petri nets and its soundness analysis, Computer Integrated Manufacturing Systems. Volume 10, Number 4, pp.381487, (2004).

4. W.M.P. Van Der Aalst, The application of Petri nets to workflow Management, The Journal of Circuits, Systems and Computers. Volume 8, Number 1, pp.21-66, (1998). 
5. A. SchoSmig and H. Rau, A Petri Net Approach for the Performance Analysis of Business Processes, Research Report Series 116, Institute of Computer Science, University of Wurzburg (1995).

6. S. Gong, J. Yang, Y. Chai, and M. Li, Business Process Modeling Based on Colored Petri nets, Information and Control. Volume 29, Number 1, pp.1-5, (2000).

7. K. Sycara and A. Pannu, Distributed Intelligent Agent, IEEE Expert. Volume12, pp.36-46, (1996).

8. J.W. Shepherdson, S.G. Thompson, and B.R. Odgers, Cross Organizational Workflow Co-ordinate by Software Agent (Oct. 19, 2000).

http//www.zurich.ibm.com/hlu/WACCworkshop/papers/Shepherdson/ 